\title{
Self-reported mental health status of pregnant women in Sweden during the COVID-19 pandemic: A cross-sectional survey
}

Ho-Fung Chung

The University of Hong Kong

\section{Ewa Andersson}

Karolinska Institutet

Hsuan-Ying Huang

The Chinese University of Hong Kong

Ganesh Acharya

Karolinska Institutet and Center for Fetal Medicine, Karolinska University Hospital

Simone Schwank ( $\nabla$ simone.schwank@ki.se )

UiT-The Arctic University of Norway

\section{Research Article}

Keywords: COVID-19, pregnancy, perinatal care, mental health, depression, anxiety, stress

Posted Date: October 6th, 2021

DOl: https://doi.org/10.21203/rs.3.rs-872040/v1

License: (9) This work is licensed under a Creative Commons Attribution 4.0 International License. Read Full License 


\section{Self-reported mental health status of pregnant women in Sweden during the COVID-19 pandemic: A cross-sectional survey}

Chung, Ho-Fung ${ }^{\#}$, Department of Psychology, The University of Hong Kong, Hong Kong, China and Department of Anthropology, The Chinese University of Hong Kong, Hong Kong, China

Ewa Andersson\# Department of Women's and Children's Health, Division of Reproductive Health, Karolinska Institutet, Stockholm, Sweden

Huang, Hsuan-Ying, Department of Anthropology, The Chinese University of Hong Kong, Hong Kong, China

Ganesh Acharya, Division of Obstetrics and Gynecology, Department of Clinical Science Intervention and Technology (CLINTEC), Karolinska Institutet and Center for Fetal Medicine, Department of Obstetrics and Gynecology, Karolinska University Hospital, Stockholm, Sweden Women's Health and Perinatology Research Group, Department of Clinical Medicine, UiT-The Arctic University of Norway, Tromsø, Norway

ORCID ID: $\underline{\text { https://orcid.org/0000-0002-1997-3107 }}$

Simone Schwank*, Division of Obstetrics and Gynecology, Department of Clinical Science Intervention and Technology (CLINTEC), Karolinska Institutet, Stockholm, Sweden.

Women's Health and Perinatology Research Group, Department of Clinical Medicine, UiT-The Arctic University of Norway, Tromsø, Norway

* Corresponding author: Schwank Simone; e-mail: simone.schwank@ki.se; phone: +46707999574; Address: Karolinska Institutet, CLINTEC, University Hospital, K56

Huddinge, 14186 Stockholm, Sweden

\# shared first authorship 


\section{Abstract}

Background: The COVID-19 pandemic has contributed to unprecedented worries and challenges for pregnant women due to social restrictions and changes in maternity care provision. We aimed to investigate the mental health impact of COVID-19 pandemic on pregnant women in Sweden and explore factors associated with poor perinatal mental health in this specific context.

Method: This was a nation-wide cross-sectional survey of pregnant women living in Sweden. Validated questionnaires were distributed through non-profit organizations' websites and social media channels from May 2020 to February 2021. Perinatal depression, anxiety, and acute stress reaction were assessed using the Edinburgh Postnatal Depression Scale (EPDS), Generalized Anxiety Disorder-7(GAD-7) and Impact Event Scale (Revised)(IES-R), respectively. Sociodemographic characteristics and self-perceived mental well-being were also obtained. Factors associated with mental health outcomes were analyzed using multivariate logistic regression model.

Results: Among a total of 522 participants, $42.5 \%(\mathrm{n}=222)$ reported depression (EPDS $\geq 13), 25.3 \%$ $(\mathrm{n}=132)$ moderate to severe anxiety (GAD-7 score $\geq 10)$, and $23.4 \%(\mathrm{n}=122)$ moderate to severe acute stress reaction (IES-R $\geq 33$ ). 27.4\% participants $(\mathrm{n}=143,27.4 \%)$ expressed concerns regarding their mental well-being during the pandemic. Pregnant mothers who had sick family members reported more severe poor mental health outcomes than those who did not (median [Interquartile range (IQR)] EPDS scores: 14.0 [8.75 - 18.0] vs 10.0 [6.0 - 14.0], $\mathrm{p}<.001$; median [IQR] GAD7 scores: 7.0 [4.0 - 12.25] vs 5.0 [3.0 - 9.0], p<.001; median (IQR) IES-R scores: 20.0 [9.0 - 38.0] vs 15.0 [7.0 - 28.0], $\mathrm{p}=.008$ ). Logistic regression analyses revealed that risk factors for poor mental health outcomes were having a sick family member with any illness, unemployment, giving birth, and experiencing an exceptional stressful life event. Having a higher educational level, younger age, and changing career during the pandemic were protective.

Conclusion: Depression and anxiety were highly prevalent among pregnant women in Sweden during the COVID-19 pandemic, indicating a need for professional mental health support for this vulnerable group of population. Unemployment was an associated risk factor whereas younger age and higher educational level were protective suggesting an important role of socio-economic factors in modulating the impact of COVID-19 pandemic on perinatal mental health.

Key words: COVID-19, pregnancy, perinatal care, mental health, depression, anxiety, stress 


\section{Background}

The consequences of the COVID-19 pandemic on global mental health are significant and its longterm impact on the global burden of disease is likely to be high. The COVID-19 pandemic has a profound impact on healthcare systems and potentially on pregnancy outcomes (Thapa et al., 2020; Yan et al., 2020). Rapid changes to the delivery of maternity health care services have occurred in many countries across the globe in response to the COVID-19 pandemic. Maternity care provisions are facing a challenge in their attempt to balance the needs and safety of pregnant women and their care providers (Palatnik \& McIntosh, 2020).

Globally, maternal and perinatal outcomes have worsened, with an increase in maternal deaths, stillbirths, ruptured ectopic pregnancies, and maternal depression (Chmielewska et al., 2021). During a situation of acute crisis, such as the COVID-19 pandemic, pregnant women are even more vulnerable than the general population in terms of susceptibility to mental health disorders (López-Morales et al., 2021) and the state of partial immune suppression (WHO, 2020). Prenatal distress and psychiatric symptomatology have also been shown to be more prevalent among pregnant women during the COVID-19 pandemic compared to the pre-pandemic period (Berthelot et al., 2020).

Mental disorders are a common cause of morbidity during pregnancy. In Sweden, the prevalence of perinatal depression pre-pandemic was $13.7 \%$ (women with high EPDS scores, i.e. $\geq 12$ ) (Rubertsson et al., 2005; Silverman et al., 2017). Swedish pre-pandemic prevalence of anxiety symptoms in early pregnancy was $15.6 \%$ (HADS-A scores $\geq 8$ ) (Rubertsson et al., 2014). Approximately, $12.0 \%$ of pregnant women report symptoms of posttraumatic stress in Sweden. Assed by post-traumatic stress symptoms (3-item). The result is not based on a diagnostic questionnaire or a clinical diagnosis (Wangel et al., 2016), while the estimated lifetime prevalence of PTSD among the general female population in Sweden is 7.4\% (Persson et al., 2020).

Sweden chose a different approach in mitigating the impact of the COVID-19 pandemic, compared to other neighboring Scandinavian countries (Engjom et al., 2021). Society remained open, while masks were not recommended until the end of 2020. Pregnant women were not considered a vulnerable group initially and until recently were excluded from being vaccinated. Husbands/partners of pregnant women have not been welcomed at the antenatal visits and in some counties not been allowed to attend the delivery. We hypothesized that such changes and adjustments in routine maternity care might have a negative impact on maternal mental health. This study aimed to investigate the impact of the COVID-19 pandemic on the mental wellbeing of pregnant women in Sweden and explore risk factors for poor perinatal mental health in this specific context.

\section{Method}




\section{Study Design and setting}

This study is part of a global survey investigating the impact of the COVID-19 pandemic on pregnant women's mental health, including perinatal depression, anxiety, and acute stress reaction. This multi-national project includes an anonymous web-based survey distributed to pregnant women in China (Hong Kong SAR and Shanghai), Norway, Sweden, Switzerland, Taiwan and all states of the United States of America (USA). The Swedish part of the survey was started in May 2020 and completed in February 2021.

Pregnant women who 1) resided in Sweden during the COVID-19 pandemic and 2) could understand Swedish could participate in the study. Exclusion criteria were 1) unconfirmed pregnancy status and 2) inability to use a web-based application to answer the survey questionnaires. The pregnant women are recruited via non-profit organizations' websites and social media channels. Via the web link, the participants were guided to the research website where they could read information about the project and answer the questionnaires anonymously if agreed to participate in the study. Informed consent was obtained from all the participants and their guardian for illiterate participants. Data were collected using Qualtrics, a widely used online survey platform for social science research.

\section{Assessment of mental health status}

The anonymous survey included assessments of symptoms of perinatal depression, anxiety and PTSD using validated instruments (Arnberg et al., 2014; Buist et al., 2011; Rubertsson et al., 2011)

Edinburgh Postnatal Depression Scale (EPDS) Depressive symptoms among pregnant mothers were assessed by EPDS (Cox et al., 1987). EPDS is a 10-item self-report scale assessing postnatal depression. Each item offers four options which are scored from 0 to 3, higher indicates more intense depressive symptoms over the past 7 days. Total score of EPDS ranges from 0 to 30 . The Swedish version of Edinburgh Postnatal Scale is well validated (Rubertsson et al., 2011) and commonly used in women mental health studies, with an optimal cut-off score of $\geq 13$. In our study, the scale demonstrated good internal consistency with a Cronbach's Alpha of .87.

Generalized Anxiety Disorder-7 (GAD7) The GAD7 is a globally used self-report scale that assesses participants' level of generalized anxiety in the past 14 days. The 7-item Likert-4 scale measures the frequency of being distressed by anxiety-related symptoms, with options ranging from not at all to nearly every day (scored from 0 to 3). Total score of GAD7 ranges from 0 to 21, higher scores indicate a higher level of generalized anxiety. The total score of GAD7 will be further categorized into severity categories of minimal (0-4), mild (5-9), moderate (10-14), and severe (15-21) anxiety. In this study, we used the Swedish version of GAD7 (Spitzer et al., 2006), which has demonstrated excellent internal consistency in previous studies.

Impact Event Scale (Revised) (IES-R). The IES-R is a 22 item self-reported measure designed to assess acute stress reactions and probable post-traumatic distress following stressful life events in the past seven days (Horowitz et al., 1979). Each item measures with a Likert-5 rating scale (from 
not at all to extremely, 0-4). Total score of IES-R ranges from 0 to 88, higher scores indicate higher levels of distress from acute stress reactions. The total scores are further categorized into categories of minimal (0-23), mild (24-32), moderate to severe concern for PTSD (33-88). In this study, IES$\mathrm{R}$ is administered as event-oriented to the covid-19 pandemic. We used the Swedish version of IES-R (Arnberg et al., 2014; Sveen et al., 2010), which has demonstrated an excellent internal consistency with a Cronbach's Alpha of .94 in this study.

Mental wellbeing and stressful life events during the pandemic. The perceived mental well-being of participants was assessed by a single 10-point Likert scale enquiring overall mental health status in the past 12 months. Participants were then asked to fill in a stressful event checklist assessing their exposures in the past one year.

\section{Statistical Analysis}

Participants' characteristics and scores obtained from mental health assessments were analyzed by descriptive statistics. Continuous variables are presented as means and standard deviations (SD), while categorical variables are presented as number (n) and percentage (\%). Associations were analyzed using Chi-squared test. The total scores of the three mental health status measurement tools (EPDS, GAD7 and IES-R) were not normally distributed, and so are presented as medians with interquartile ranges (IQR). Non-parametric Mann-Whitney U test was applied to compare the severity of symptoms between families with sick family members (including COVID-19 and other illnesses) and those without sick family members. Associations between mental health outcomes (depression and anxiety, acute stress reactions) and demographic characteristics of participants were estimated by univariate and multivariate logistic regression, and are presented as odds ratios (ORs) with 95\% Confidence intervals (CIs). The statistical significance was set at a $p$-value of $<0.05$. All statistical analyses were performed with the Statistical Package for the Social Science (SPSS) version 26 (IBM® SPSS $®$ Statistics).

\section{Results}

\section{Demographic Characteristics of the Participants}

A total of 522 pregnant women completed the questionnaire; $31.0 \%(n=162)$ of whom had at least one sick family member. The sociodemographic characteristics of the participants, their selfperceived overall mental health status, and stressful event exposure are presented in Table 1. The mean age of the participants was $30.81( \pm 4.39)$ years. Most women lived in cohabitation with a partner $(55.0 \%)$ or were married $(42.0 \%)$ and the majority $(n=301,57.7 \%)$ were working 40 hours a week. Two third of the participants $(n=317,60.7 \%)$ were with a bachelor's degree or above.

Table 1 Sociodemographic characteristics of participants $(\mathrm{N}=522)$

\begin{tabular}{|c|c|c|c|c|c|}
\hline \multirow[b]{3}{*}{ Variables } & \multirow[b]{3}{*}{ Mean, SD } & \multicolumn{4}{|c|}{ No. $(\%)$} \\
\hline & & \multirow[b]{2}{*}{ Total } & \multicolumn{3}{|c|}{ Family Health Status } \\
\hline & & & Families & Families & $P$ value* \\
\hline
\end{tabular}




\begin{tabular}{|c|c|c|c|c|c|c|}
\hline & & & & \multirow{2}{*}{\multicolumn{2}{|c|}{$\begin{array}{cc}\text { with sick } & \text { without sick } \\
\text { members } & \text { members } \\
\end{array}$}} & \\
\hline & & & & & & \\
\hline \multicolumn{3}{|l|}{ Overall } & $522(100)$ & $162(31.0)$ & $360(69.0)$ & \\
\hline \multirow[t]{5}{*}{ Age } & & $30.81(4.39)$ & & & & \\
\hline & $18-25$ & & $53(10.2)$ & $18(11.1)$ & $35(9.7)$ & 689 \\
\hline & $26-30$ & & $203(38.9)$ & $65(40.1)$ & $138(38.3)$ & \\
\hline & $31-40$ & & $257(49.2)$ & $75(46.3)$ & $182(50.6)$ & \\
\hline & $>40$ & & $9(1.7)$ & $4(2.5)$ & $5(1.4)$ & \\
\hline \multicolumn{7}{|c|}{ Civil Status } \\
\hline & Single & & $3(0.6)$ & $1(0.6)$ & $2(0.6)$ & .671 \\
\hline & Married & & $219(42.0)$ & $69(42.6)$ & $150(41.7)$ & \\
\hline & Cohabitating & & $287(55.0)$ & $87(53.7)$ & $200(55.6)$ & \\
\hline & $\begin{array}{l}\text { in a } \\
\text { relationship }\end{array}$ & & $12(2.3)$ & $4(2.5)$ & $8(2.2)$ & \\
\hline & Divorced & & $1(0.2)$ & $1(0.6)$ & $0(0)$ & \\
\hline \multicolumn{7}{|c|}{ Working Hours } \\
\hline & $\begin{array}{l}<40 \text { hours per } \\
\text { week }\end{array}$ & & $177(33.9)$ & $59(36.4)$ & $118(32.8)$ & .170 \\
\hline & $\begin{array}{l}40 \text { hours per } \\
\text { week }\end{array}$ & & $301(57.7)$ & $85(52.5)$ & $216(60.0)$ & \\
\hline & $\begin{array}{l}>40 \text { hours per } \\
\text { week }\end{array}$ & & $44(8.4)$ & $18(11.1)$ & $26(7.2)$ & \\
\hline \multicolumn{7}{|c|}{ Monthly Family Income } \\
\hline & $<40000 \mathrm{SEK}$ & & $177(34.6)$ & $44(28.2)$ & $133(37.4)$ & $.029 *$ \\
\hline & $40000 \mathrm{SEK}$ & & $212(41.4)$ & $78(50.0)$ & $134(37.6)$ & \\
\hline & $>40000 \mathrm{SEK}$ & & $123(24.0)$ & $34(21.8)$ & $89(25.0)$ & \\
\hline \multicolumn{7}{|c|}{ Educational Level } \\
\hline & No education & & $3(0.6)$ & $0(0)$ & $3(0.8)$ & .806 \\
\hline & $\begin{array}{l}\text { 9-year } \\
\text { mandatory } \\
\text { education }\end{array}$ & & $10(1.9)$ & $4(2.5)$ & $6(1.7)$ & \\
\hline & High school & & $108(20.7)$ & $33(20.4)$ & $75(20.8)$ & \\
\hline & $\begin{array}{l}\text { Professional } \\
\text { education }\end{array}$ & & $84(16.1)$ & $30(18.5)$ & $54(15.0)$ & \\
\hline & Bachelor & & $167(32.0)$ & $48(29.6)$ & $119(33.1)$ & \\
\hline & Masters & & $140(26.8)$ & $44(27.2)$ & $96(26.7)$ & \\
\hline & Doctoral & & $10(1.9)$ & $3(1.9)$ & $7(1.9)$ & \\
\hline \multirow[t]{3}{*}{$\begin{array}{l}\text { Overall } \\
\text { Status }(\end{array}$} & tal Health & $4.84(1.90)$ & & & & \\
\hline & Healthy & & $379(72.6)$ & $102(63.0)$ & $277(76.9)$ & $.001 * *$ \\
\hline & $\begin{array}{l}\text { Worsened } \\
\text { Mental Health } \\
(\geq 6)\end{array}$ & & $143(27.4)$ & $60(37.0)$ & $83(23.1)$ & \\
\hline
\end{tabular}

Stressfull event Exposure 


\begin{tabular}{lllll} 
Pregnant & $470(90.0)$ & $142(87.7)$ & $328(91.1)$ & .222 \\
Giving Birth & $58(11.1)$ & $16(9.9)$ & $42(11.7)$ & .547 \\
Marriage & $37(7.1)$ & $12(7.4)$ & $25(6.9)$ & .849 \\
Divorce & $0(0)$ & $0(0)$ & $0(0)$ & N/A \\
Death in & $58(11.1)$ & $22(13.6)$ & $36(10.0)$ & .229 \\
families & & & & \\
Changing & $76(14.6)$ & $21(13.0)$ & $55(15.3)$ & .488 \\
Career & & & & \\
Exams & $30(5.8)$ & $10(6.2)$ & $20(5.6)$ & .779 \\
Unemployment & $41(7.9)$ & $16(9.9)$ & $25(6.9)$ & .249 \\
Others & $153(29.3)$ & $38(23.5)$ & $115(31.9)$ & $\mathbf{. 0 4 9 *}$ \\
\hline
\end{tabular}

\section{Mental Health Status of the Pregnant Mothers and Severity Categories}

The overall prevalence of probable depression for pregnant women during the pandemic (EPDS $\geq$ 13) was $42.5 \%(n=222)$. One fourth $(n=132,25.3 \%)$ of Swedish pregnant women participating in the study displayed moderate to severe generalized anxiety symptoms (GAD-7 $\geq 10$ ). 23.4\% $(n=122)$ of the participants had moderate to severe concern for PTSD (IES-R $\geq 33$ ).

Women whose families had sick members with any illness in the past 12 months reported experiencing more depression, anxiety and acute stress reactions (probable depression: 93(57.4\%) vs 129 (35.8\%), $p<.001$; moderate to severe anxiety: 58 (35.8\%) vs $74(20.5 \%), p<.001$; moderate to severe concern for probable PTSD: $47(29.4 \%)$ vs $75(21.1 \%), p<.001)$ (See Table 2).

\section{Scores of Measurements}

The total median scores (IQR) on the EPDS for depression, GAD7 for anxiety, and IES-R for acute stress reaction among women were $11.0(7.0-16.0), 6.0(3.0-10.0)$, and $16.0(7.0-31.0)$ respectively. Similar to findings in severity categories of women, women whose families had sick members had higher scores for depression, anxiety and acute stress reaction compared with those who did not (median EPDS scores: 14.0 (8.75 - 18.0) vs $10.0(6.0$ - 14.0), $\mathrm{p}<.001$; median GAD7 scores: $7.0(4.0-12.25)$ vs $5.0(3.0-9.0), \mathrm{p}<.001$; median IES-R scores: 20.0 (9.0 - 38.0) vs 15.0 $(7.0-28.0), p=.004)($ See Table 3).

\section{Factors Associated with mental health status of pregnant women}

Multivariate logistic regression analyses were performed to investigate factors associated with the mental health status of pregnant women during the pandemic. Having sick family members increased the odds of depression by 3 times (aOR 2.950, 95\% CI $1.933-4.502, p<.001$ ), anxiety by 2.5 times (aOR: $2.548,95 \%$ CI $1.615-4.022, p<.001$ ), acute stress reaction by $60 \%$ (aOR $1.604,95 \%$ CI $1.004-2.560, \mathrm{p}=.048)$, and perceived worsening in mental health by 2 times (aOR $1.951,95 \%$ CI $1.405-3.391, \mathrm{p}=.001)$. Unemployment increased the odds of depression by 2.5 times (aOR 2.499, 95\% CI 1.140 - 5.477, $p=.022)$ and acute stress reaction by 2.8 times (aOR $2.812,95 \%$ CI $1.362-5.805, p=.005)$. Encountering an exceptional stressful life event increased the odds of depression (aOR 1.879, $1.215-2.908, p=.005$ ), anxiety (aOR 2.299, 95\% CI $1.426-$ $3.708, p=.001)$, and so did the perceived worsening of mental health during the pandemic (aOR $1.951,95 \%$ CI $1.233-3.090, p=.004)$. Anticipation of giving birth during the pandemic increased 
the odds of acute stress reaction by 2.5 times (aOR 2.459, 95\% CI $1.323-4.570, p=.004$ ). (See Table 4-7).

Having a higher level of education (i.e. having a bachelor degree or above) decreased the odds of all three mental health outcomes (depression: aOR .761, 95\% CI .637 - .911, $p=.003$; anxiety: aOR .821, 95\% CI .676 - .997, $p=.047$; acute stress reaction: aOR .515, 95\% CI $.315-.841, p=.008$ ) Women in the age group of 26 to 30 or 31 to 40 were less likely to develop anxiety symptoms (aOR .464, 95\% CI .229 - .942, $p=.034$; aOR .360, 95\% CI .168 - .772, $p=.009$ ) and perceived worsening of mental health (aOR .495, 95\% CI .246 - .996, $p=.049$; aOR .360, 95\% CI . $170-.760$, $p=.007$ ). Being at the age of 31 to 40 years decreased the odds of depression (aOR $.458,95 \%$ CI .216 - .972, $p=.042$ ). Changing career reduced the odds of acute stress reaction (aOR .446, 95\% CI .202 - .986, $p=.046$ ). (See Table 4-7) 
Table. 2 Self-reported mental health status and severity of depression, anxiety, and acute stress in total study cohort and subgroups

\begin{tabular}{|c|c|c|c|c|c|c|c|}
\hline \multirow[t]{2}{*}{ Severity Category } & \multirow[t]{2}{*}{$\begin{array}{l}\text { Total, } \\
(\%)\end{array}$} & \multirow[t]{2}{*}{ Mean (SD) } & \multicolumn{2}{|c|}{$\begin{array}{l}\text { Families with sick } \\
\text { members }\end{array}$} & \multicolumn{2}{|c|}{$\begin{array}{l}\text { Families without sick } \\
\text { members }\end{array}$} & \multirow[t]{2}{*}{$\begin{array}{l}P \\
\text { value }\end{array}$} \\
\hline & & & No. $(\%)$ & Mean (SD) & No. $(\%)$ & Mean (SD) & \\
\hline \multicolumn{8}{|l|}{ EPDS, depression symptoms } \\
\hline Total cohort & $522(100.0)$ & $11.63(6.28)$ & $162(31.0)$ & $13.47(6.51)$ & $360(69.0)$ & $10.81(6.01)$ & \\
\hline Normal & $300(57.5)$ & & $69(42.6)$ & & $231(64.2)$ & & $<.001$ \\
\hline $\begin{array}{l}\text { Probable Depression (scores } \geq \\
\text { 13) }\end{array}$ & $222(42.5)$ & & $93(57.4)$ & & $129(35.8)$ & & \\
\hline \multicolumn{8}{|l|}{ GAD7, Anxiety } \\
\hline Total cohort & $522(100.0)$ & $6.90(5.28)$ & $162(31.0)$ & $8.25(5.64)$ & $360(69.0)$ & $6.30(5.00)$ & \\
\hline Normal (0-4) & $199(38.1)$ & & $48(29.6)$ & & $151(41.9)$ & & $<.001$ \\
\hline Mild (5-9) & $191(36.6)$ & & $56(34.6)$ & & $135(37.5)$ & & \\
\hline Moderate (10-14) & $71(13.6)$ & & $28(17.3)$ & & 43 (11.9) & & \\
\hline Severe $(15-21)$ & $61(11.7)$ & & $30(18.5)$ & & $31(8.6)$ & & \\
\hline \multicolumn{8}{|l|}{ IES-R, Acute Stress symptoms } \\
\hline Total cohort & $516(100.0)$ & $21.06(17.60)$ & $160(31.0)$ & $24.43(19.27)$ & $356(69.0)$ & $19.55(16.61)$ & \\
\hline Normal (0-23) & $345(66.9)$ & & $98(61.3)$ & & $247(69.4)$ & & .051 \\
\hline Mild (24-32) & $49(9.5)$ & & $15(9.4)$ & & $34(9.6)$ & & \\
\hline $\begin{array}{l}\text { Moderate to Severe concern for } \\
\text { PTSD }(33-88)\end{array}$ & $122(23.4)$ & & $47(29.4)$ & & $75(21.1)$ & & \\
\hline
\end{tabular}


Table. 3 Scores of Mental health Measurements in Total Cohort and Subgroups

\begin{tabular}{|c|c|c|c|c|c|c|c|}
\hline \multirow[t]{2}{*}{ Scale } & \multirow[t]{2}{*}{$\begin{array}{l}\text { Total score, } \\
\text { Median }\end{array}$} & \multirow[t]{2}{*}{ IQR } & \multicolumn{2}{|c|}{ Families with sick members } & \multicolumn{2}{|c|}{$\begin{array}{l}\text { Families without sick } \\
\text { members }\end{array}$} & \multirow[t]{2}{*}{$\begin{array}{l}P \\
\text { value }\end{array}$} \\
\hline & & & $\begin{array}{l}\text { Total score, } \\
\text { Median }\end{array}$ & IQR & $\begin{array}{l}\text { Total } \\
\text { Median }\end{array}$ & IQR & \\
\hline $\begin{array}{l}\text { EPDS, } \\
\text { Symptoms }\end{array}$ & 11.0 & $7.0-16.0$ & 14.0 & $8.75-18.0$ & 10.0 & $6.0-14.0$ & $<.001$ \\
\hline GAD7, Anxiety Symptoms & 6.0 & $3.0-10.0$ & 7.0 & $4.0-12.25$ & 5.0 & $3.0-9.0$ & $<.001$ \\
\hline $\begin{array}{l}\text { IES-R, Acute Stress } \\
\text { Reaction }\end{array}$ & 16.0 & $7.0-31.0$ & 20.0 & $9.0-38.0$ & 15.0 & $7.0-28.0$ & .008 \\
\hline
\end{tabular}

Aberration: EPDS, 10-item Edinburgh Postnatal Depression Scale; GAD-7, 7-item Generalized Anxiety Disorder; IES-R, 22-item Impact of Event Scale-Revised; IQR, Interquartile Range 
Table. 4 Factors associated with depressive symptoms (EPDS $\geq 13$ ) among pregnant women

\begin{tabular}{|c|c|c|c|c|c|c|}
\hline & cOR & $95 \% \mathrm{CI}$ & $p$ & aOR & $95 \% \mathrm{CI}$ & $p$ \\
\hline \multicolumn{7}{|l|}{ Age } \\
\hline $18-25$ & Ref & Ref & & Ref & Ref & \\
\hline $26-30$ & .394 & $.209-.741$ & $.004 * *$ & .514 & $.251-1.052$ & .069 \\
\hline $31-40$ & .307 & $.165-.571$ & $<.001 * *$ & .458 & $.216-.972$ & $.042 *$ \\
\hline$>40$ & .257 & $.057-1.150$ & .076 & .313 & $.060-1.645$ & .170 \\
\hline \multicolumn{7}{|l|}{ Working Hours } \\
\hline$<40$ hours per week & Ref & Ref & & Ref & Ref & \\
\hline 40 hours per week & .663 & $.456-.966$ & $.032 *$ & .873 & $.566-1.347$ & .539 \\
\hline$>40$ hours per week & .882 & $.455-1.710$ & .710 & 1.069 & $.508-2.250$ & .860 \\
\hline \multicolumn{7}{|l|}{ Monthly Family Income } \\
\hline$<40000 \mathrm{SEK}$ & Ref & Ref & & Ref & Ref & \\
\hline $40000 \mathrm{SEK}$ & 1.266 & $.847-1.894$ & .250 & .765 & $.474-1.235$ & .273 \\
\hline$>40000 \mathrm{SEK}$ & .748 & $.465-1.204$ & .232 & .881 & $.524-1.482$ & .634 \\
\hline \multicolumn{7}{|l|}{ Educational Level } \\
\hline Bachelor or above & .441 & $.308-.632$ & $<.001 * *$ & .761 & $.637-.911$ & $.003 * *$ \\
\hline \multicolumn{7}{|l|}{ Family Health Status } \\
\hline With sick family members & 2.414 & $1.653-3.525$ & $<.001 * *$ & 2.950 & $1.933-4.502$ & $<.001 * *$ \\
\hline \multicolumn{7}{|l|}{ Pregnancy } \\
\hline Yes & 1.321 & $.730-2.390$ & .358 & 1.685 & $.870-3.265$ & .122 \\
\hline \multicolumn{7}{|l|}{ Giving Birth } \\
\hline Yes & 1.518 & $.878-2.623$ & .135 & 1.760 & $.961-3.222$ & .067 \\
\hline \multicolumn{7}{|l|}{ Marriage } \\
\hline Yes & 1.305 & $.668-2.549$ & .436 & 1.230 & $.583-2.592$ & .587 \\
\hline \multicolumn{7}{|l|}{ Death in families } \\
\hline Yes & 1.299 & $.752-2.245$ & .349 & 1.301 & $.714-2.371$ & .389 \\
\hline \multicolumn{7}{|l|}{ Changing Career } \\
\hline Yes & .663 & $.399-1.104$ & .114 & .769 & $.432-1.371$ & .374 \\
\hline \multicolumn{7}{|l|}{ Exams } \\
\hline Yes & .660 & $.303-1.440$ & .297 & .860 & $.356-2.079$ & .738 \\
\hline \multicolumn{7}{|l|}{ Unemployment } \\
\hline Yes & 3.606 & $1.796-7.240$ & $<.001 * *$ & 2.499 & $1.140-5.477$ & $.022 *$ \\
\hline \multicolumn{7}{|l|}{ Others } \\
\hline Yes & 1.508 & $1.032-2.203$ & $.034 *$ & 1.879 & $1.215-2.908$ & $.005 * *$ \\
\hline
\end{tabular}

$* p<.05, * * p<.01$ 
Table. 5 Factors associated with anxiety symptoms (GAD7 $\geq 10$ ) among pregnant women

\begin{tabular}{|c|c|c|c|c|c|c|}
\hline & cOR & $95 \% \mathrm{CI}$ & $p$ & aOR & $95 \% \mathrm{CI}$ & $p$ \\
\hline \multicolumn{7}{|l|}{ Age } \\
\hline $18-25$ & Ref & Ref & & Ref & Ref & \\
\hline $26-30$ & .427 & $.229-.798$ & $.008 * *$ & .464 & $.229-.942$ & $.034 *$ \\
\hline $31-40$ & .321 & $.173-.597$ & $<.001 * *$ & .360 & $.168-.772$ & $.009 * *$ \\
\hline$>40$ & .151 & $.018-1.294$ & .085 & .184 & $.019-1.753$ & .141 \\
\hline \multicolumn{7}{|l|}{ Working Hours } \\
\hline$<40$ hours per week & Ref & Ref & & Ref & Ref & \\
\hline 40 hours per week & .895 & $.584-1.373$ & .613 & 1.042 & $.637-1.705$ & .869 \\
\hline$>40$ hours per week & 1.329 & $.648-2.724$ & .437 & 1.348 & $.595-3.052$ & .474 \\
\hline \multicolumn{7}{|l|}{ Monthly Family Income } \\
\hline$<40000 \mathrm{SEK}$ & Ref & Ref & & Ref & Ref & \\
\hline $40000 \mathrm{SEK}$ & 1.459 & $.911-2.336$ & .116 & .887 & $.509-1.545$ & .671 \\
\hline$>40000 \mathrm{SEK}$ & 1.387 & $.809-2.378$ & .234 & 1.635 & $.915-2.922$ & .097 \\
\hline \multicolumn{7}{|l|}{ Educational Level } \\
\hline Bachelor or above & .554 & $.372-.825$ & $.004 * *$ & .821 & $.676-.997$ & $.047 *$ \\
\hline \multicolumn{7}{|l|}{ Family Health Status } \\
\hline With sick family members & 2.155 & $1.430-3.249$ & $<.001 * *$ & 2.548 & $1.615-4.022$ & $.000 * *$ \\
\hline \multicolumn{7}{|l|}{ Pregnancy } \\
\hline Yes & 1.292 & $.644-2.594$ & .471 & 1.672 & $.788-3.549$ & .180 \\
\hline \multicolumn{7}{|l|}{ Giving Birth } \\
\hline Yes & 1.382 & $.762-2.505$ & .287 & 1.731 & $.895-3.347$ & .103 \\
\hline \multicolumn{7}{|l|}{ Marriage } \\
\hline Yes & 1.273 & $.611-2.653$ & .520 & 1.166 & $.524-2.593$ & .707 \\
\hline \multicolumn{7}{|l|}{ Death in families } \\
\hline Yes & 1.258 & $.688-2.301$ & .455 & 1.220 & $.643-2.313$ & .543 \\
\hline \multicolumn{7}{|l|}{ Changing Career } \\
\hline Yes & 1.065 & $.612-1.853$ & .823 & 1.319 & $.713-2.440$ & .378 \\
\hline \multicolumn{7}{|l|}{ Exams } \\
\hline Yes & .726 & $.290-1.817$ & .494 & 1.117 & $.412-3.028$ & .828 \\
\hline \multicolumn{7}{|l|}{ Unemployment } \\
\hline Yes & 2.254 & $1.170-4.343$ & $.015 *$ & 1.601 & $.757-3.389$ & .218 \\
\hline \multicolumn{7}{|l|}{ Others } \\
\hline Yes & 1.699 & $1.119-2.580$ & $.013 *$ & 2.299 & $1.426-3.708$ & $.001 * *$ \\
\hline
\end{tabular}

${ }^{*} p<.05, * * p<.01$ 
Table. 6 Factors associated with acute stress reaction (IES-R $\geq 33$ ) among pregnant women

\begin{tabular}{|c|c|c|c|c|c|c|}
\hline & cOR & $95 \% \mathrm{CI}$ & $p$ & aOR & $95 \% \mathrm{CI}$ & $p$ \\
\hline \multicolumn{7}{|l|}{ Age } \\
\hline $18-25$ & Ref & Ref & & Ref & Ref & \\
\hline $26-30$ & .488 & $.258-.923$ & $.027 *$ & .733 & $.358-1.500$ & .395 \\
\hline $31-40$ & .368 & $.195-.692$ & $.002 * *$ & .644 & $.301-1.380$ & .258 \\
\hline$>40$ & .762 & $.172-3.385$ & .721 & 1.094 & $.212-5.642$ & .914 \\
\hline \multicolumn{7}{|l|}{ Working Hours } \\
\hline$<40$ hours per week & Ref & Ref & & Ref & Ref & \\
\hline 40 hours per week & .798 & $.517-1.231$ & .307 & 1.146 & $.699-1.879$ & .589 \\
\hline$>40$ hours per week & .850 & $.388-1.860$ & .684 & 1.091 & $.451-2.640$ & .847 \\
\hline \multicolumn{7}{|l|}{ Monthly Family Income } \\
\hline$<40000 \mathrm{SEK}$ & Ref & Ref & & Ref & Ref & \\
\hline $40000 \mathrm{SEK}$ & 1.362 & $.858-2.162$ & .189 & .897 & $.523-1.539$ & .693 \\
\hline$>40000 \mathrm{SEK}$ & .663 & $.366-1.203$ & .177 & .748 & $.396-1.412$ & .370 \\
\hline \multicolumn{7}{|l|}{ Educational Level } \\
\hline Bachelor or above & .419 & $.277-.633$ & $<.001 * *$ & .515 & $.315-.841$ & $.008 * *$ \\
\hline \multicolumn{7}{|l|}{ Family Health Status } \\
\hline With sick family members & 1.558 & $1.019-2.384$ & $.041 *$ & 1.604 & $1.004-2.560$ & $.048 *$ \\
\hline \multicolumn{7}{|l|}{ Pregnancy } \\
\hline Yes & 1.036 & $.525-2.044$ & .919 & 1.091 & $.522-2.283$ & .816 \\
\hline \multicolumn{7}{|l|}{ Giving Birth } \\
\hline Yes & 2.383 & $1.346-4.219$ & $.003 * *$ & 2.459 & $1.323-4.570$ & $.004 * *$ \\
\hline \multicolumn{7}{|l|}{ Marriage } \\
\hline Yes & 1.041 & $.477-2.271$ & .919 & .954 & $.407-2.237$ & .914 \\
\hline \multicolumn{7}{|l|}{ Death in families } \\
\hline Yes & .825 & $.422-1.614$ & .575 & .870 & $.426-1.773$ & .701 \\
\hline \multicolumn{7}{|l|}{ Changing Career } \\
\hline Yes & .396 & $.191-.820$ & $.013 *$ & .446 & $.202-.986$ & $.046 *$ \\
\hline \multicolumn{7}{|l|}{ Exams } \\
\hline Yes & .480 & $.164-1.403$ & .180 & .809 & $.259-2.529$ & .715 \\
\hline \multicolumn{7}{|l|}{ Unemployment } \\
\hline Yes & 3.483 & $1.817-6.674$ & $<.001 * *$ & 2.812 & $1.362-5.805$ & $.005 * *$ \\
\hline \multicolumn{7}{|l|}{ Others } \\
\hline Yes & 1.259 & $.813-1.950$ & .301 & 1.335 & $.821-2.171$ & .244 \\
\hline
\end{tabular}

${ }^{*} p>.05,{ }^{* *} p>.01$ 
Table. 7 Factors associated with worsen mental health (Global rating scale $\geq 6$ ) among pregnant women

\begin{tabular}{|c|c|c|c|c|c|c|}
\hline & cOR & $95 \% \mathrm{CI}$ & $p$ & aOR & $95 \% \mathrm{CI}$ & $p$ \\
\hline \multicolumn{7}{|l|}{ Age } \\
\hline $18-25$ & Ref & Ref & & Ref & Ref & \\
\hline $26-30$ & .437 & $.235-.813$ & $.009 * *$ & .495 & $.246-.996$ & $.049 *$ \\
\hline $31-40$ & .319 & $.173-.590$ & $<.001 * *$ & .360 & $.170-.760$ & $.007 * *$ \\
\hline$>40$ & .896 & $.216-3.711$ & .880 & .980 & $.207-4.641$ & .980 \\
\hline \multicolumn{7}{|l|}{ Working Hours } \\
\hline$<40$ hours per week & Ref & Ref & & Ref & Ref & \\
\hline 40 hours per week & .610 & $.404-.922$ & $.019 *$ & .701 & $.440-1.117$ & .135 \\
\hline$>40$ hours per week & 1.172 & $.588-2.337$ & .651 & 1.294 & $.602-2.780$ & .509 \\
\hline \multicolumn{7}{|l|}{ Monthly Family Income } \\
\hline$<40000 \mathrm{SEK}$ & Ref & Ref & & Ref & Ref & \\
\hline $40000 \mathrm{SEK}$ & 1.162 & $.747-1.809$ & .506 & .785 & $.465-1.325$ & .364 \\
\hline$>40000 \mathrm{SEK}$ & .792 & $.463-1.354$ & .394 & .940 & $.530-1.668$ & .832 \\
\hline \multicolumn{7}{|l|}{ Educational Level } \\
\hline Bachelor or above & .555 & $.376-.819$ & $.003 * *$ & .754 & $.470-1.210$ & .242 \\
\hline \multicolumn{7}{|l|}{ Family Health Status } \\
\hline With sick family members & 1.963 & $1.313-2.935$ & $.001 * *$ & 2.183 & $1.405-3.391$ & $.001 * *$ \\
\hline \multicolumn{7}{|l|}{ Pregnancy } \\
\hline Yes & 1.027 & $.539-1.958$ & .936 & 1.149 & $.572-2.310$ & .696 \\
\hline \multicolumn{7}{|l|}{ Giving Birth } \\
\hline Yes & 1.220 & $.675-2.208$ & .510 & 1.361 & $.716-2.587$ & .347 \\
\hline \multicolumn{7}{|l|}{ Marriage } \\
\hline Yes & 1.902 & $.957-3.779$ & .067 & 1.860 & $.893-3.873$ & .097 \\
\hline \multicolumn{7}{|l|}{ Death in families } \\
\hline Yes & 1.459 & $.817-2.604$ & .201 & 1.503 & $.811-2.787$ & .196 \\
\hline \multicolumn{7}{|l|}{ Changing Career } \\
\hline Yes & .670 & $.372-1.207$ & .182 & .800 & $.420-1.525$ & .498 \\
\hline \multicolumn{7}{|l|}{ Exams } \\
\hline Yes & .648 & $.259-1.169$ & .353 & .831 & $.307-2.251$ & .716 \\
\hline \multicolumn{7}{|l|}{ Unemployment } \\
\hline Yes & 1.784 & $.923-3.450$ & .085 & 1.134 & $.536-2.397$ & .743 \\
\hline \multicolumn{7}{|l|}{ Others } \\
\hline Yes & 1.643 & $1.091-2.473$ & $.017 *$ & 1.951 & $1.233-3.090$ & $.004 * *$ \\
\hline
\end{tabular}

${ }^{*} p>.05, * * p>.01$ 


\section{Discussion}

Women's healthcare is often adversely affected by humanitarian disasters (Yerger et al., 2020). Our findings highlight the importance of planning robust maternity services in any emergency response, in line with the findings of a recent review on the impact of the COVID-19 pandemic on pregnant women (Chmielewska et al., 2021). Pregnant women in Sweden appeared to have suffered more than prior to the pandemic, based on our finding of substantially increased prevalence of perinatal depression, anxiety, and acute stress reaction. Similar to the present study, one of the first studies conducted under the COVID-19 pandemic in China has shown that pregnant women had significantly higher rates of depressive symptoms $(26.0 \%$ vs $29.6 \%, \mathrm{P}=0.02)$ than women assessed before the pandemic was declared (Wu et al., 2020)

In our study, the prevalence of depressive symptoms among pregnant women in Sweden was exceptionally high, both in comparison with pre-pandemic prevalence studies from Sweden (Silverman et al., 2017), neighboring Scandinavian countries (Silverman et al., 2017), as well as internationally (Cameron et al., 2020; Torales et al., 2020). This result stands in contrast to the expectations that in an open, non-confined society, such as Sweden, pregnant women would have better mental well-being compared to societies with prolonged isolation during the pandemic.

The inclination of "adopting social distancing measures" is often thought to be positively associated with worsened mental health during the pandemic. However, it has proven not to be the case in a study in New York City (Silverman et al., 2020). Silverman et al. found that in women with low socioeconomic status, who are most vulnerable for prenatal mood disruption, the social restrictions reduced their mental health problems. A decrease in symptomatology was also found in a recent Gallup Panel 2021 (Gallup, 2021) reporting a decrease in worry after restrictions were put in place, compared to earlier in the pandemic. Therefore, loose social restrictions leading to worries for disease transmission could be a potential explanation of why the Swedish pregnant reported an exceptionally high? prevalence of mental health problems.

The prevalence of depression (EPDS scores of $\geq 13$ ) among Swedish women in pregnancy during the pandemic was high at $42.5 \%$. Compared to the mean EPDS score acquired in a Swedish study 12\% (mean: 5.0, $\mathrm{n}=110$ ) before the pandemic (Massoudi et al., 2016), we found it to be doubled during the pandemic at $11.63(\mathrm{n}=522)$. Similarly, the pre-pandemic prevalence of anxiety symptoms in pregnant women residing in Sweden (HADS-A scores $\geq 8$ during early pregnancy) was $15.6 \%$ (Rubertsson et al., 2014), while during the pandemic the point prevalence (GAD7 scores $\geq 10$ ) has increased to $25.3 \%$. However, this needs to be interpreted with caution as the women participating in our study were at variable stages in their pregnancy.

With regards to acute stress reaction, the estimated lifetime prevalence of acute stress and in severe conditions of probable PTSD among women in Sweden is 7.4\% (Frans et al., 2005) using diagnostic procedures and the PTSD Checklist (PCL), a series of posttraumatic stress scales to assess acute stress and PTSD developed by Persson (Persson et al., 2020). Current PTSD, based 
on diagnostic criteria and severity, was reported by 4.1 percent (95\% CI 2.8-5.8) of the pregnant women(Persson et al., 2020) .

Perrson et al found in their study conducted in 2017-2018, that a majority of pregnant women with PTSD experienced violence, and expressed a fear of childbirth (Persson et al., 2020). Both domestic violence and fear of childbirth have increased globally, as a consequence of the COVID19 pandemic (Berthelot et al., 2020). Although we did not inquire about domestic violence in our survey, these results are important to consider, in relation to the present study. In our study, the prevalence of moderate to severe concern for probable PTSD among the Swedish pregnant women during the pandemic was $23.4 \%$. The rise in acute stress reaction and PTSD symptoms has also been shown in previous research during the SARS and MERS outbreaks (Lee et al., 2007; Lee et al., 2019; Lee et al., 2018; Mak et al., 2010; Park et al., 2020). Similar results occurred after the 9/11 terrorist attack (Rousseau et al., 2015) and the Holocaust (Bowers \& Yehuda, 2016; Yehuda et al., 2005; Yehuda et al., 1998). The clinically concerning acute stress in pregnant women may impact their fetuses (Doyle et al., 2015). Chronic stress may cause an epigenetic change in the placenta, facilitating cortisol to pass through easier, which can result in the fetus brain maturing faster, and upon birth being hypervigilant (Monk, 2016; Monk et al., 2016; O’Connor et al., 2016). Beside the potential impact on the fetus, there is a risk of increase in number of women experiencing fear of labor and childbirth, which is important for healthcare professionals to be aware of to avoid unnecessary operative deliveries and associated complications.

When we compared participants, who were from families that had sick members and those who were not, we found that poor family health status had increased the odds of having perinatal mental health issues. Unemployment and exceptionally stressful encounters were also associated with an increasing likelihood of exhibiting probable perinatal mental disorders during the COVID-19 pandemic. This is not surprising as lower socio-economic status is known to be associated with higher risk of perinatal mental health problems. Rectifying long-standing health inequalities in the societies is equally important while making swift responses to the impact of the pandemic.

In our study, women in the age groups of 26 to 30 and 31 to 40 years showed significantly lower levels of anxiety. It could be related to the fact that in these age groups, most mothers have stable families, economic stability, and good social networks.

\section{Strengths and limitations}

Our study is one of the first in Sweden to evaluate mental health of pregnant women during COVID-19 pandemic. Data collection took place form May 2020 to May 2021, initiating the data collection during the peak of the pandemic in Sweden. However, it is not without limitations. Convenient sampling method limits the generalization of our findings, and the cross-sectional design did not allow to study the trends over a longer time and differences in mental health status of pregnant women during different stages of gestation. The study population of 522 pregnant women, potentially reduces the generalizability of the results to the general population of Swedish pregnant women. Potential for misinterpretation of questions by participants cannot be excluded 
as the survey was self-administered online. Prevalence of anxiety, depression, and stress in pregnant women were found to be very high, and future studies with larger sample size are needed to evaluate the prevalence of perinatal mental health disorders due to the COVID-19 pandemic in Sweden.

The consequences on global mental health due to COVID-19 will be significant and cause longterm impact on the global burden of disease. Mental health problems cannot not be overlooked in this regard. It is important to understand now what women need to cope with and navigate their pregnancies during this pandemic. It is important that women receive reliable information and professional from their healthcare providers rather than getting conflicting advice in the social media as a primary source of information.

The high prevalence of perinatal mental health problems found in this study suggests the need for a systematic approach to screening pregnant women and providing professional support to those at high risk. Providing psychological first aid and counselling are essential during a pandemic. It helps in reducing the psychological distress and promoting adaptive coping strategies to deal with the situation short and long term (Humer et al., 2021; Moreno et al., 2020). Web-based psychosocial support can be an invaluable resource to bridge the treatment gap of perinatal mental health problems (Schwank et al., 2020), which has clearly increased during the global pandemic. Results from pre-pandemic research on the effect of internet-based support for perinatal mental health care services in Sweden, have shown significantly lower levels of depressive symptoms post treatment. Pregnancy adapted internet based psychosocial support for antenatal depression is therefore feasible, acceptable and efficacious (Forsell et al., 2017).

\title{
Conclusion
}

Depression and anxiety were highly prevalent among pregnant women in Sweden during the COVID-19 pandemic indicating a need for professional mental health support for this vulnerable group of population. Unemployment was an associated risk factor whereas younger age and higher educational level were protective suggesting an important role of socio-economic factors in modulating the impact of COVID-19 pandemic on perinatal mental health.

\author{
Abberations \\ aOR: Adjusted Odds Ratio \\ CI: Confidence Interval \\ cOR: Crude Odds Ratio \\ COVID-19: Coronavirus Disease 2019 \\ EPDS: Edinburgh Postnatal Depression Scale \\ GAD7: Generalized Anxiety Disorder 7 \\ HADS-A: Hospital Anxiety and Depression Scale - Anxiety
}


IES-R: Impact of Event Scale -Revised

IQR: Interquartile Range

MERS: Middle East respiratory syndrome

PTSD: Post-traumatic stress disorder

SARS: Severe acute respiratory syndrome

SEK: Swedish Krona

Please check author instructions, I think reference list should go before the Declarations.

\section{Declarations}

\section{Ethical approval}

Informed consent was obtained from all the participants and their guardian for illiterate participants. All methods were performed in accordance with the relevant guidelines and regulations of the Helsinki Declaration. The study was approved by the Swedish Ethical Review Authority, Etikprövnings Myndigheten (ref: Dnr Ö 21-2020/3.1; date of approval: 2020.05.18). Each participant consented to the study before participating in the survey.

\section{Consent for publication}

Not applicable

\section{Availability of data and materials}

The dataset generated and analyzed in the current study is not publicly available. However, anonymized data can be made available from the corresponding author on reasonable request.

\section{Competing interests}

Authors have no competing interest to declare in relation to this article.

\section{Funding}

This project was funded by Swiss National Science Foundation (SNSF) (P2SKP3_187728), The Swedish Foundation for International Cooperation in Research and Higher Education (STINT), and the Chinese American Psychoanalytic Alliance (CAPA). The funding agencies did not have any part in the design, data collection and analysis or reporting of the results.

\section{Author contributions}

Conception and design of study: All coauthors H.-F.C., S.E.S., E.A., H.-Y.H, G.A. contributed, discussed, agreed, and wrote the research protocol.

Acquisition of data: H.-F.C., S.E.S., E.A. 
Analysis and/or interpretation of data: All the coauthors contributed H.-F.C.., S.E.S., E.A., H.-Y.H, G.A.

Drafting the manuscript: H.-F.C. and S.E.S.

Revising the manuscript critically for important intellectual content: All coauthors contributed H.F.C., S.E.S., E.A., H.-Y.H, G.A.

Approval of the version of the manuscript to be published H.-F.C., S.E.S., E.A., H.-Y.H, G.A.

\section{Acknowledgements}

None

\section{References}

1. Thapa SB, Mainali A, Schwank SE, Acharya G. Maternal mental health in the time of the COVID-19 pandemic. Acta obstetricia et gynecologica Scandinavica. 2020;99(7):817-8.

2. Yan H, Ding Y, Guo W. Mental Health of Pregnant and Postpartum Women During the Coronavirus Disease 2019 Pandemic: A Systematic Review and Meta-Analysis. Frontiers in psychology. 2020;11:617001-.

3. Palatnik A, McIntosh JJ. Protecting Labor and Delivery Personnel from COVID-19 during the Second Stage of Labor. American journal of perinatology. 2020;37(8):854-6.

4. Chmielewska B, Barratt I, Townsend R, Kalafat E, van der Meulen J, Gurol-Urganci I, et al. Effects of the COVID-19 pandemic on maternal and perinatal outcomes: a systematic review and meta-analysis. The Lancet global health. 2021.

5. López-Morales H, Del-Valle MV, Andrés ML, Gelpi Trudo R, Canet-Juric L, Urquijo S. Longitudinal study on prenatal depression and anxiety during the COVID-19 pandemic. Archives of women's mental health. 2021.

6. WHO. Naming the coronavirus disease (COVID-19) and the virus that causes it. World Health Organization; 2020.

7. Berthelot N, Lemieux R, Garon-Bissonnette J, Drouin-Maziade C, Martel É, Maziade M. Uptrend in distress and psychiatric symptomatology in pregnant women during the coronavirus disease 2019 pandemic. Acta obstetricia et gynecologica Scandinavica. 2020;99(7):848-55.

8. Rubertsson C, Wickberg B, Gustavsson P, Rådestad I. Depressive symptoms in early pregnancy, two months and one year postpartum-prevalence and psychosocial risk factors in a national Swedish sample. Archives of women's mental health. 2005;8(2):97-104.

9. Silverman ME, Reichenberg A, Savitz DA, Cnattingius S, Lichtenstein P, Hultman CM, et al. The risk factors for postpartum depression: A population-based study. Depression and anxiety. 2017;34(2):178-87.

10. Rubertsson C, Hellström J, Cross M, Sydsjö G. Anxiety in early pregnancy: prevalence and contributing factors. Archives of women's mental health. 2014;17(3):221-8.

11. Wangel A-M, Ryding EL, Schei B, Östman M, Lukasse M. Emotional, physical, and sexual abuse and the association with symptoms of depression and posttraumatic stress in a multi-ethnic pregnant population in southern Sweden. Sexual \& reproductive healthcare. 2016;9:7-13. 
12. Persson A, Lindmark S, Petersson K, Gabriel E, Thorsell M, Lindström K, et al. Potentially traumatic events, fear of childbirth and posttraumatic stress disorder during pregnancy in Stockholm, Sweden: A cross-sectional study. Sexual \& reproductive healthcare. 2020;25:100516-.

13. Engjom H, Aabakke AJM, Klungsøyr K, Svanvik T, Äyräs O, Jonasdottir E, et al. COVID-19 in pregnancy-characteristics and outcomes of pregnant women admitted to hospital because of SARS-CoV-2 infection in the Nordic countries. Acta obstetricia et gynecologica Scandinavica. 2021.

14. Rubertsson C, Börjesson K, Berglund A, Josefsson A, Sydsjö G. The Swedish validation of Edinburgh Postnatal Depression Scale (EPDS) during pregnancy. Nordic journal of psychiatry. 2011;65(6):414-8.

15. Buist A, Gotman N, Yonkers KA. Generalized anxiety disorder: Course and risk factors in pregnancy. Journal of Affective Disorders. 2011;131(1-3):277-83.

16. Arnberg FK, Michel P-O, Johannesson KB. Properties of Swedish posttraumatic stress measures after a disaster. Journal of anxiety disorders. 2014;28(4):402-9.

17. Cox JL, Holden JM, Sagovsky R. Detection of postnatal depression. Development of the 10- item Edinburgh Postnatal Depression Scale. The British journal of psychiatry : the journal of mental science. 1987;150:782.

18. Spitzer RL, Kroenke K, Williams JBW, Löwe B. A Brief Measure for Assessing Generalized Anxiety Disorder: The GAD-7. Archives of Internal Medicine. 2006;166(10):10927.

19. Horowitz M, Wilner N, Alvarez W. Impact of Event Scale: a measure of subjective stress. Psychosomatic medicine. 1979;41(3):209-18.

20. Sveen J, Low A, Dyster-Aas J, Ekselius L, Willebrand M, Gerdin B. Validation of a Swedish version of the Impact of Event Scale-Revised (IES-R) in patients with burns. Journal of Anxiety Disorders. 2010;24(6):618-22.

21. Yerger P, Jalloh M, Coltart CEM, King C. Barriers to maternal health services during the Ebola outbreak in three West African countries: a literature review. BMJ global health. 2020;5(9):e002974.

22. Wu Y, Zhang C, Liu H, Duan C, Li C, Fan J, et al. Perinatal depressive and anxiety symptoms of pregnant women during the coronavirus disease 2019 outbreak in China. American journal of obstetrics and gynecology. 2020;223(2):240.e1-.e9.

23. Cameron EE, Joyce KM, Delaquis CP, Reynolds K, Protudjer JLP, Roos LE. Maternal psychological distress \& mental health service use during the COVID-19 pandemic. Journal of affective disorders. 2020;276:765-74.

24. Torales J, O'Higgins M, Castaldelli-Maia JM, Ventriglio A. The outbreak of COVID-19 coronavirus and its impact on global mental health. International Journal of Social Psychiatry. 2020;66(4):317-20.

25. Silverman ME, Medeiros C, Burgos L. Early pregnancy mood before and during COVID-19 community restrictions among women of low socioeconomic status in New York City: a preliminary study. Archives of women's mental health. 2020;23(6):779-82.

26. Coronavirus Pandemic [Internet]. 2021 [cited 16.08.2021]. Available from: https://news.gallup.com/poll/308222/coronavirus-pandemic.aspx.

27. Rousseau C, Jamil U, Bhui K, Boudjarane M. Consequences of 9/11 and the war on terror on children's and young adult's mental health: A systematic review of the past 10 years. Clinical child psychology and psychiatry. 2015;20(2):173-93. 
28. Humer E, Pieh C, Probst T, Kisler I-M, Schimböck W, Schadenhofer P. Telephone Emergency Service 142 (TelefonSeelsorge) during the COVID-19 Pandemic: Cross-Sectional Survey among Counselors in Austria. International journal of environmental research and public health. 2021;18(5):2228.

29. Moreno C, Wykes T, Galderisi S, Nordentoft M, Crossley N, Jones N, et al. How mental health care should change as a consequence of the COVID-19 pandemic. The Lancet Psychiatry. 2020;7(9):813-24.

30. Forsell E, Bendix M, Holländare F, von Schultz BS, Nasiell J, Blomdahl-Wetterholm M, et al. Internet delivered Cognitive Behavior Therapy for Antenatal Depression: A Randomised Controlled Trial. Journal of affective disorders. 2017;221:56-64.

Arnberg, F. K., Michel, P.-O., \& Johannesson, K. B. (2014). Properties of Swedish posttraumatic stress measures after a disaster. Journal of anxiety disorders, 28(4), 402-409. https://doi.org/10.1016/j.janxdis.2014.02.005

Berthelot, N., Lemieux, R., Garon - Bissonnette, J., Drouin - Maziade, C., Martel, É., \& Maziade, M. (2020). Uptrend in distress and psychiatric symptomatology in pregnant women during the coronavirus disease 2019 pandemic. Acta obstetricia et gynecologica Scandinavica, 99(7), 848-855. https://doi.org/10.1111/aogs.13925

Buist, A., Gotman, N., \& Yonkers, K. A. (2011). Generalized anxiety disorder: Course and risk factors in pregnancy. Journal of Affective Disorders, 131(1-3), 277-283. https://doi.org/10.1016/j.jad.2011.01.003

Cameron, E. E., Joyce, K. M., Delaquis, C. P., Reynolds, K., Protudjer, J. L. P., \& Roos, L. E. (2020). Maternal psychological distress \& mental health service use during the COVID19 pandemic. Journal of affective disorders, 276, 765-774. https://doi.org/10.1016/j.jad.2020.07.081

Chmielewska, B., Barratt, I., Townsend, R., Kalafat, E., van der Meulen, J., Gurol-Urganci, I., . . . Khalil, A. (2021). Effects of the COVID-19 pandemic on maternal and perinatal outcomes: a systematic review and meta-analysis. The Lancet global health. https://doi.org/10.1016/S2214-109X(21)00079-6

Cox, J. L., Holden, J. M., \& Sagovsky, R. (1987). Detection of postnatal depression. Development of the 10- item Edinburgh Postnatal Depression Scale. The British journal of psychiatry : the journal of mental science, 150, 782.

Engjom, H., Aabakke, A. J. M., Klungsøyr, K., Svanvik, T., Äyräs, O., Jonasdottir, E., . . . Krebs, L. (2021). COVID-19 in pregnancy-characteristics and outcomes of pregnant women admitted to hospital because of SARS-CoV-2 infection in the Nordic countries. Acta obstetricia et gynecologica Scandinavica. https://doi.org/10.1111/aogs.14160

Forsell, E., Bendix, M., Holländare, F., von Schultz, B. S., Nasiell, J., Blomdahl-Wetterholm, M., ... Kaldo, V. (2017). Internet delivered Cognitive Behavior Therapy for Antenatal Depression: A Randomised Controlled Trial. Journal of affective disorders, 221, 56-64. https://doi.org/10.1016/j.jad.2017.06.013

Frans, O., Rimmo, P. A., Aberg, L., \& Fredrikson, M. (2005). Trauma exposure and posttraumatic stress disorder in the general population. Acta psychiatrica Scandinavica, 111(4), 291-290. https://doi.org/10.1111/j.1600-0447.2004.00463.x

Gallup. (2021). Coronavirus Pandemic 
Horowitz, M., Wilner, N., \& Alvarez, W. (1979). Impact of Event Scale: a measure of subjective stress. Psychosomatic medicine, 41(3), 209-218. https://doi.org/10.1097/00006842197905000-00004

Humer, E., Pieh, C., Probst, T., Kisler, I.-M., Schimböck, W., \& Schadenhofer, P. (2021). Telephone Emergency Service 142 (TelefonSeelsorge) during the COVID-19 Pandemic: Cross-Sectional Survey among Counselors in Austria. International journal of environmental research and public health, 18(5), 2228. https://doi.org/10.3390/ijerph18052228

López-Morales, H., Del-Valle, M. V., Andrés, M. L., Gelpi Trudo, R., Canet-Juric, L., \& Urquijo, S. (2021). Longitudinal study on prenatal depression and anxiety during the COVID-19 pandemic. Archives of women's mental health. https://doi.org/10.1007/s00737-021-01152-1

Massoudi, P., Hwang, C. P., \& Wickberg, B. (2016). Fathers' depressive symptoms in the postnatal period: Prevalence and correlates in a population-based Swedish study. Scandinavian journal of public health, 44(7), 688-694. https://doi.org/10.1177/1403494816661652

Moreno, C., Wykes, T., Galderisi, S., Nordentoft, M., Crossley, N., Jones, N., . . Arango, C. (2020). How mental health care should change as a consequence of the COVID-19 pandemic. The Lancet. Psychiatry, 7(9), 813-824. https://doi.org/10.1016/S22150366(20)30307-2

Palatnik, A., \& McIntosh, J. J. (2020). Protecting Labor and Delivery Personnel from COVID-19 during the Second Stage of Labor. American journal of perinatology, 37(8), 854-856. https://doi.org/https://dx.doi.org/10.1055/s-0040-1709689

Persson, A., Lindmark, S., Petersson, K., Gabriel, E., Thorsell, M., Lindström, K., . . . Magnusson, A. (2020). Potentially traumatic events, fear of childbirth and posttraumatic stress disorder during pregnancy in Stockholm, Sweden: A cross-sectional study. Sexual \& reproductive healthcare, $25,100516-100516$. https://doi.org/10.1016/j.srhc.2020.100516

Rousseau, C., Jamil, U., Bhui, K., \& Boudjarane, M. (2015). Consequences of 9/11 and the war on terror on children's and young adult's mental health: A systematic review of the past 10 years. Clinical child psychology and psychiatry, 20(2), 173-193. https://doi.org/10.1177/1359104513503354

Rubertsson, C., Börjesson, K., Berglund, A., Josefsson, A., \& Sydsjö, G. (2011). The Swedish validation of Edinburgh Postnatal Depression Scale (EPDS) during pregnancy. Nordic journal of psychiatry, 65(6), 414-418. https://doi.org/10.3109/08039488.2011.590606

Rubertsson, C., Hellström, J., Cross, M., \& Sydsjö, G. (2014). Anxiety in early pregnancy: prevalence and contributing factors. Archives of women's mental health, 17(3), 221-228. https://doi.org/10.1007/s00737-013-0409-0

Rubertsson, C., Wickberg, B., Gustavsson, P., \& Rådestad, I. (2005). Depressive symptoms in early pregnancy, two months and one year postpartum-prevalence and psychosocial risk factors in a national Swedish sample. Archives of women's mental health, 8(2), 97-104. https://doi.org/10.1007/s00737-005-0078-8

Silverman, M. E., Medeiros, C., \& Burgos, L. (2020). Early pregnancy mood before and during COVID-19 community restrictions among women of low socioeconomic status in New York City: a preliminary study. Archives of women's mental health, 23(6), 779-782. https://doi.org/https://dx.doi.org/10.1007/s00737-020-01061-9 
Silverman, M. E., Reichenberg, A., Savitz, D. A., Cnattingius, S., Lichtenstein, P., Hultman, C. M., .. . Sandin, S. (2017). The risk factors for postpartum depression: A population based study. Depression and anxiety, 34(2), 178-187. https://doi.org/10.1002/da.22597

Spitzer, R. L., Kroenke, K., Williams, J. B. W., \& Löwe, B. (2006). A Brief Measure for Assessing Generalized Anxiety Disorder: The GAD-7. Archives of Internal Medicine, 166(10), 1092-1097. https://doi.org/10.1001/archinte.166.10.1092

Sveen, J., Low, A., Dyster-Aas, J., Ekselius, L., Willebrand, M., \& Gerdin, B. (2010). Validation of a Swedish version of the Impact of Event Scale-Revised (IES-R) in patients with burns. Journal of Anxiety Disorders, 24(6), 618-622. https://doi.org/10.1016/j.janxdis.2010.03.021

Thapa, S. B., Mainali, A., Schwank, S. E., \& Acharya, G. (2020). Maternal mental health in the time of the COVID - 19 pandemic. Acta obstetricia et gynecologica Scandinavica, 99(7), 817-818. https://doi.org/10.1111/aogs.13894

Torales, J., O’Higgins, M., Castaldelli-Maia, J. M., \& Ventriglio, A. (2020). The outbreak of COVID-19 coronavirus and its impact on global mental health. International Journal of Social Psychiatry, 66(4), 317-320. https://doi.org/10.1177/0020764020915212

Wangel, A.-M., Ryding, E. L., Schei, B., Östman, M., \& Lukasse, M. (2016). Emotional, physical, and sexual abuse and the association with symptoms of depression and posttraumatic stress in a multi-ethnic pregnant population in southern Sweden. Sexual \& reproductive healthcare, 9, 7-13. https://doi.org/10.1016/j.srhc.2016.04.003

WHO. (2020). Naming the coronavirus disease (COVID-19) and the virus that causes it. World Health Organization Retrieved from https://www.who.int/emergencies/diseases/novelcoronavirus-2019/technical-guidance/naming-the-coronavirus-disease-(covid-2019)-andthe-virus-that-causes-it

Wu, Y., Zhang, C., Liu, H., Duan, C., Li, C., Fan, J., . . . Huang, H.-F. (2020). Perinatal depressive and anxiety symptoms of pregnant women during the coronavirus disease 2019 outbreak in China. American journal of obstetrics and gynecology, 223(2), 240.e241-240.e249. https://doi.org/https://dx.doi.org/10.1016/j.ajog.2020.05.009

Yan, H., Ding, Y., \& Guo, W. (2020). Mental Health of Pregnant and Postpartum Women During the Coronavirus Disease 2019 Pandemic: A Systematic Review and MetaAnalysis. Frontiers in psychology, 11, 617001-617001. https://doi.org/10.3389/fpsyg.2020.617001

Yerger, P., Jalloh, M., Coltart, C. E. M., \& King, C. (2020). Barriers to maternal health services during the Ebola outbreak in three West African countries: a literature review. BMJ global health, 5(9), e002974. https://doi.org/10.1136/bmjgh-2020-002974 